Research Article

\title{
Polarization and DOA Estimation Based on Dual-Polarized Conformal Array
}

\author{
Chao Liu $(\mathbb{D}$, Shuai Xiang $(\mathbb{D}$, Liangfeng Xu $(\mathbb{D}$, and Zhengfei Fang $\mathbb{( D}$ \\ Department of Communication Engineering, Hefei University of Technology, Hefei, Anhui 230009, China \\ Correspondence should be addressed to Chao Liu; disneyl@hfut.edu.cn
}

Received 8 May 2019; Accepted 28 August 2019; Published 18 September 2019

Academic Editor: N. Nasimuddin

Copyright (c) 2019 Chao Liu et al. This is an open access article distributed under the Creative Commons Attribution License, which permits unrestricted use, distribution, and reproduction in any medium, provided the original work is properly cited.

\begin{abstract}
A dual-polarized multiple signal classification (DP-MUSIC) algorithm is presented to estimate the arrival directions and polarizations for a dual-polarized conformal array. Each polarization signal is decomposed into two orthogonal polarization components, which are considered to be a pair of coherent signals coming from the same direction but different polarization. The polarization parameters are modeled as the equivalent coherence coefficients of the orthogonal polarization components. Then, the method of decoherence can be used to decouple the information of polarization states and signal angles. After that, the direction of arrival (DOA) and polarization parameters can be estimated by the DP-MUSIC algorithm. Moreover, the angles of incident direction are re-estimated, which greatly improves the accuracy of DOA estimation. The Cramer-Rao bound (CRB) is derived and the effectiveness of the proposed algorithm is verified by Monte Carlo simulations.
\end{abstract}

\section{Introduction}

Conformal array is an antenna array that conforms to the surface of the carrier. Conformal antennas have been widely applied in the area of radar, navigation, radio communications, and satellite applications with their special advantages [1]. Super-resolution direction finding is one of the most important research directions. However, due to the inconsistency of the polarization characteristics of the conformal array, the information of polarization and direction of arrival (DOA) are coupled with each other, which brings new problems to the estimation of the parameters.

The multiple signal classification (MUSIC) algorithm was proposed by Schmidt to estimate the DOA of the signal in [2]. In [3], the MUSIC-based DOA estimation algorithm incorporating the oblique projection was proposed. In [4], a method for estimating the arrival direction of array interpolation based on compressed sensing was proposed. However, these algorithms leave out of consideration the effects of polarization of antenna elements.

Polarization is a problem that cannot be ignored. Therefore, the polarization sensitivity of array to incident signals needs to be considered in DOA estimation. In [5], the estimation of signal parameters via rotational invariance techniques (ESPRIT) was proposed to estimate the DOA and polarization parameters of incoming plane waves with a uniform linear array. In $[6,7]$, the polarization smoothing algorithm (PSA) was proposed to estimate arrival directions and polarizations for a coherent source. This method requires the steering vector of the array to be written as the Kronecker product of the spatial steering vector and the polarized vector. Actually, only planar array can satisfy this relationship, and conformal array does not have these characteristics because of the inconsistency of the polarization of the elements.

In [8], a blind polarization DOA estimation method was proposed by using three pairs of special subarrays for conical conformal array. In [9], based on the fourth-order cumulant of array measurements and special array design structure, a blind DOA estimation algorithm was proposed for the conformal array. However, they require that the conformal array contains linear subarrays. Therefore, these methods are not applicable to the arbitrary conformal array structure. The aforementioned works for directions and polarizations are studied based on the single-polarized array. In $[10,11]$, the performance of the single-polarized and dual-polarized 
antenna array was analyzed and compared. It is possible to conclude that the performance of a dual-polarized antenna array is significantly better than that of a single-polarized array. In [12], DOA and polarization estimation for unequal power sources was proposed based on reconstructed noise subspace. However, this method is not suitable for conformal arrays. In [13], a blind polarization DOA estimation method was proposed based on the dual feed conformal array for polarization diversity signals. But this method is only applicable to orthogonal polarization signals and does not involve DOA estimation of general polarization signals.

In this paper, a dual-polarized multiple signal classification (DP-MUSIC) method is presented based on the dual-polarized conformal array. Each polarization signal is decomposed into two orthogonal polarization components, which are considered to be a pair of coherent signals. The polarization parameters are modeled as the coherence coefficients of each pair of polarization components. And then, decoupling of polarization and DOA can be converted to the decoherence problem. Since the conventional decoherence technique cannot be applied to the conformal array, it is firstly projected onto a uniform planar array. And then, the forward-backward smoothing technique is adopted to solve the coherence problem. After that, the DOA and polarization parameters can be estimated. DPMUSIC-based spectrum is constructed for the DOA estimation. And the corresponding polarization parameters can be estimated by using the estimated DOA. Additionally, DOA is re-estimated by using the original received data and the estimated polarization parameters to further improve the DOA estimation accuracy. The Cramer-Rao bound $(\mathrm{CRB})$ is derived to verify the performance of the algorithm. Finally, the validity of the proposed algorithm is demonstrated by simulation experiments.

\section{Signal Model}

Consider an ideal dual-polarized conformal array with $N$ elements shown in Figure 1. The coupling between antenna elements and polarization components is not considered, and the two feed points of the dual-polarized antenna excite and receive their polarization components independently [14]. $O(x, y, z)$ is the global coordinate system of the conformal array, and $O_{i}^{\prime}\left(x^{\prime}, y^{\prime}, z^{\prime}\right)$ is the local coordinate system of the $i$ th array element. $\theta$ and $\phi$ represent the elevation and azimuth of the incident signal, respectively. $\theta_{i}^{\prime}$ and $\phi_{i}^{\prime}$ are the associated elevation and azimuth in the $i$ th local coordinate system.

Assume that the two feed points of the antenna receive the signals of $\theta$-polarization and the $\phi$-polarization component, respectively. In order to conveniently synthesis the far field pattern of the conformal array, the components of all the elements need to be unified into the same coordinate system to count their contributions to the whole conformal array radiation. The mapping angle $\left(\theta_{i}^{\prime}, \phi_{i}^{\prime}\right)$ of the direction $(\theta, \phi)$ in the $i$ th local coordinate system can be calculated by the following formula:

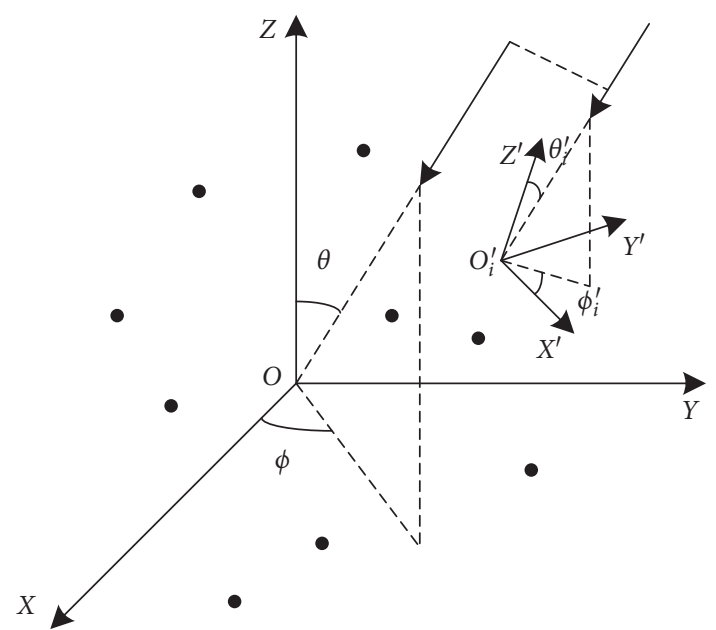

FIgURE 1: Conformal array coordinate diagram.

$$
\left[\begin{array}{c}
\sin \theta_{i}^{\prime} \cos \phi_{i}^{\prime} \\
\sin \theta_{i}^{\prime} \sin \phi_{i}^{\prime} \\
\cos \theta_{i}^{\prime}
\end{array}\right]=\mathbf{R}_{\mathrm{gli}}\left[\begin{array}{c}
\sin \theta \cos \phi \\
\sin \theta \sin \phi \\
\cos \theta
\end{array}\right]
$$

where $\mathbf{R}_{\mathrm{gli}}$ signifies the Euler rotation matrix from global to local of $i$ th element $[15,16]$. In order to calculate the mapping component of the signal in the local coordinate system conveniently, it is transformed into a Cartesian coordinate system.

$$
\left\{\begin{array}{l}
s_{x}(t)=-s_{\theta}(t) \cos \theta \cos \phi-s_{\phi}(t) \sin \phi \\
s_{y}(t)=-s_{\theta}(t) \cos \theta \sin \phi+s_{\phi}(t) \cos \phi \\
s_{z}(t)=s_{\theta}(t) \sin \theta
\end{array}\right.
$$

Define the transform matrix $\mathbf{T}_{\mathrm{sc}}(\theta, \phi)$ from spherical coordinates to Cartesian coordinates in the direction $(\theta, \phi)$.

$$
\mathbf{T}_{\mathrm{sc}}(\theta, \phi)=\left[\begin{array}{cc}
-\cos \theta \cos \phi & -\sin \phi \\
-\cos \theta \sin \phi & \cos \phi \\
\sin \theta & 0
\end{array}\right] .
$$

Equation (2) can be expressed as

$$
\left[\begin{array}{c}
s_{x}(t) \\
s_{y}(t) \\
s_{z}(t)
\end{array}\right]=\mathbf{T}_{\mathrm{sc}}(\theta, \phi)\left[\begin{array}{l}
s_{\theta}(t) \\
s_{\phi}(t)
\end{array}\right]
$$

The mapping components of the signal in the local coordinate system can be expressed as

$$
\begin{aligned}
& {\left[\begin{array}{c}
s_{x^{\prime}}(t) \\
s_{y^{\prime}}(t) \\
s_{z^{\prime}}(t)
\end{array}\right]=\mathbf{R}_{\mathrm{gli}}\left[\begin{array}{c}
s_{x}(t) \\
s_{y}(t) \\
s_{z}(t)
\end{array}\right],} \\
& {\left[\begin{array}{c}
s_{i \theta^{\prime}}(t) \\
s_{i \phi^{\prime}}(t)
\end{array}\right]=\mathbf{T}_{\mathrm{csi}}\left(\theta_{i}^{\prime}, \phi_{i}^{\prime}\right)\left[\begin{array}{c}
s_{x^{\prime}}(t) \\
s_{y^{\prime}}(t) \\
s_{z^{\prime}}(t)
\end{array}\right],}
\end{aligned}
$$


where $\mathbf{T}_{\mathrm{csi}}\left(\theta_{i}^{\prime}, \phi_{i}^{\prime}\right)$ represents the transform matrix of the $i$ th element from Cartesian coordinates to spherical coordinates in the direction $\left(\theta_{i}^{\prime}, \phi_{i}^{\prime}\right)$.

$$
\mathbf{T}_{\text {csi }}\left(\theta_{i}^{\prime}, \phi_{i}^{\prime}\right)=\left[\begin{array}{ccc}
0 & 0 & \frac{-1}{\sin \theta_{i}^{\prime}} \\
-\sin \phi_{i}^{\prime} & \cos \phi_{i}^{\prime} & 0
\end{array}\right] .
$$

Then, the electric field components of the signal in the $\theta$ and $\phi$ directions of the $i$ th element can be expressed as

$$
\left[\begin{array}{l}
s_{i \theta^{\prime}}(t) \\
s_{i \phi^{\prime}}(t)
\end{array}\right]=\mathbf{T}_{\mathrm{csi}}\left(\theta_{i}^{\prime}, \phi_{i}^{\prime}\right) \mathbf{R}_{\mathrm{gli}} \mathbf{T}_{\mathrm{sc}}(\theta, \phi)\left[\begin{array}{l}
s_{\theta}(t) \\
s_{\phi}(t)
\end{array}\right] .
$$

Define the transformation matrix of the $i$ th array element $\mathbf{T}_{i}(\theta, \phi)=\mathbf{T}_{\mathrm{csi}}\left(\theta_{i}^{\prime}, \phi_{i}^{\prime}\right) \mathbf{R}_{\mathrm{gli}} \mathbf{T}_{\mathrm{sc}}(\theta, \phi)$. The mapped components of the signal on the whole array can be expressed as

$$
\left[\begin{array}{c}
s_{1 \theta^{\prime}}(t) \\
s_{1 \phi^{\prime}}(t) \\
\vdots \\
s_{N \theta^{\prime}}(t) \\
s_{N \phi^{\prime}}(t)
\end{array}\right]=\left[\begin{array}{c}
\mathbf{T}_{1}(\theta, \phi) \\
\vdots \\
\mathbf{T}_{N}(\theta, \phi)
\end{array}\right]\left[\begin{array}{c}
s_{\theta}(t) \\
s_{\phi}(t)
\end{array}\right] .
$$

Without considering the noise, the received data of array can be expressed as

$$
\left[\begin{array}{c}
x_{1 \theta^{\prime}}(t) \\
x_{1 \phi^{\prime}}(t) \\
\vdots \\
x_{N \theta^{\prime}}(t) \\
x_{N \phi^{\prime}}(t)
\end{array}\right]=\left[\begin{array}{c}
g_{1 \theta^{\prime}}\left(\theta_{1}^{\prime}, \phi_{1}^{\prime}\right) \mathbf{a}_{1}(\theta, \phi) s_{1 \theta^{\prime}}(t) \\
g_{1 \phi^{\prime}}\left(\theta_{1}^{\prime}, \phi_{1}^{\prime}\right) \mathbf{a}_{1}(\theta, \phi) s_{1 \phi^{\prime}}(t) \\
\vdots \\
g_{N \theta^{\prime}}\left(\theta_{N}^{\prime}, \phi_{N}^{\prime}\right) \mathbf{a}_{N}(\theta, \phi) s_{N \theta^{\prime}}(t) \\
g_{N \phi^{\prime}}\left(\theta_{N}^{\prime}, \phi_{N}^{\prime}\right) \mathbf{a}_{N}(\theta, \phi) s_{N \phi^{\prime}}(t)
\end{array}\right],
$$

where $g_{i \theta^{\prime}}\left(\theta^{\prime}, \phi^{\prime}\right)$ and $g_{i \phi^{\prime}}\left(\theta^{\prime}, \phi^{\prime}\right)$ are $\theta$-polarized and $\phi$-polarized components of the $i$ th antenna element pattern in the local coordinate system, respectively.

$$
\mathbf{a}(\theta, \phi)=\left[e^{-j k \mathbf{r}_{1} \mathbf{v}}, \cdots, e^{-j k \mathbf{r}_{N} \mathbf{v}}\right]^{T},
$$

where $\mathbf{r}_{i}=\left[x_{i}, y_{i}, z_{i}\right], i=1,2, \cdots, N$ is the position vector of $i$ th array element in the global coordinate system. $\mathbf{v}=[\sin \theta \cos \phi, \sin \theta \sin \phi, \cos \theta]^{T}$ is the unit direction vector of $(\theta, \phi) . k=2 \pi / \lambda$ is the wavenumber, where $\lambda$ is the wavelength of the incoming signals. The symbol " $[\cdot]^{T}$ " means the transpose of matrix. Define

$$
\begin{aligned}
& \mathbf{G}(\theta, \phi)= \operatorname{diag}\left\{g_{1 \theta^{\prime}}\left(\theta_{1}^{\prime}, \phi_{1}^{\prime}\right), g_{1 \phi^{\prime}}\left(\theta_{1}^{\prime}, \phi_{1}^{\prime}\right), \cdots,\right. \\
&\left.g_{N \theta^{\prime}}\left(\theta_{N}^{\prime}, \phi_{N}^{\prime}\right), g_{N \phi^{\prime}}\left(\theta_{N}^{\prime}, \phi_{N}^{\prime}\right)\right\}, \\
& \mathbf{T}(\theta, \phi)=\left[\begin{array}{cccc}
\mathbf{T}_{1}(\theta, \phi) & \mathbf{0} & \ldots & \mathbf{0} \\
\mathbf{0} & \mathbf{T}_{2}(\theta, \phi) & \ldots & \mathbf{0} \\
\vdots & \vdots & \ddots & \vdots \\
\mathbf{0} & \mathbf{0} & \ldots & \mathbf{T}_{N}(\theta, \phi)
\end{array}\right] .
\end{aligned}
$$

Equation (9) can be expressed as

$$
\mathbf{x}(t)=\mathbf{G}(\theta, \phi) \mathbf{T}(\theta, \phi)\left(\mathbf{a}(\theta, \phi) \otimes \mathbf{I}_{2}\right)\left[\begin{array}{l}
s_{\theta}(t) \\
s_{\phi}(t)
\end{array}\right],
$$

where $\mathbf{I}_{2}$ is the $2 \times 2$ identity matrix.

$$
\begin{aligned}
\widetilde{\mathbf{a}}(\theta, \phi) & =\mathbf{G}(\theta, \phi) \mathbf{T}(\theta, \phi)\left(\mathbf{a}(\theta, \phi) \otimes \mathbf{I}_{2}\right) \\
& =\left[\widetilde{\mathbf{a}}_{\theta}(\theta, \phi), \widetilde{\mathbf{a}}_{\phi}(\theta, \phi)\right],
\end{aligned}
$$

where $\widetilde{\mathbf{a}}_{\theta}(\theta, \phi)$ and $\widetilde{\mathbf{a}}_{\phi}(\theta, \phi)$ represent the $2 N \times 1$ equivalent steering vector for the $\theta$-polarized and $\phi$-polarized components coming from the direction $(\theta, \phi)$, respectively. The symbol " $\otimes$ " means the Kronecker product.

Although the previous derivation is based on the linear polarization model, the model can also be applied to a conformal array of arbitrary dual-polarized antennas. The steering vector of arbitrary dual-polarized conformal array can be written as

$$
\widetilde{\mathbf{a}}(\theta, \phi)=\mathbf{G}(\theta, \phi) \mathbf{T}(\theta, \phi)\left(\mathbf{a}(\theta, \phi) \otimes \mathbf{L}^{-T}\right),
$$

where $\mathbf{L}$ is a linear transformation matrix between different polarizations. For the dual-polarized antennas consisting of circular polarization, the linear transformation matrix is

$$
\mathbf{L}=\left[\begin{array}{cc}
\frac{1}{\sqrt{2}} & \frac{-j}{\sqrt{2}} \\
\frac{1}{\sqrt{2}} & \frac{j}{\sqrt{2}}
\end{array}\right] .
$$

If one dual-polarized component-antenna consists of an $\theta$-polarized antenna, whereas another dual-polarized component-antenna consists of a right-hand circular antenna, the linear transformation matrix is

$$
\mathbf{L}=\left[\begin{array}{cc}
1 & 0 \\
\frac{1}{\sqrt{2}} & \frac{j}{\sqrt{2}}
\end{array}\right]
$$

Without loss of generality, the conformal array model of $\theta$-polarization and $\phi$-polarization is adopted in the following derivation and analysis.

Consider an incident signal $s(t)$ with arbitrary polarization, and it is decomposed into two orthogonal polarization signals as $s_{\theta}(t)=\sin \gamma e^{j \eta} s(t)$ and $s_{\phi}(t)=\cos \gamma s(t)$, respectively. Suppose that there are $K$ narrow-band independent sources in the far field, and the received data of array can be expressed as

$$
\begin{aligned}
x(t) & =\sum_{i=1}^{K} \widetilde{\mathbf{a}}_{\theta}\left(\theta_{i}, \phi_{i}\right) s_{i \theta}(t)+\sum_{i=1}^{K} \widetilde{\mathbf{a}}_{\phi}\left(\theta_{i}, \phi_{i}\right) s_{i \phi}(t)+\mathbf{n}(t) \\
& =\sum_{i=1}^{K}\left[\widetilde{\mathbf{a}}_{\theta}\left(\theta_{i}, \phi_{i}\right) \sin \gamma_{i} e^{j \eta_{i}}+\widetilde{\mathbf{a}}_{\phi}\left(\theta_{i}, \phi_{i}\right) \cos \gamma_{i}\right] s_{i}(t)+\mathbf{n}(t) .
\end{aligned}
$$

Define the block diagonal matrix consisting of polarization parameters as 


$$
\begin{gathered}
\mathbf{P}(\gamma, \eta)=\left[\begin{array}{ccc}
\mathbf{p}\left(\gamma_{1}, \eta_{1}\right) & \cdots & \mathbf{0} \\
\vdots & \ddots & \vdots \\
\mathbf{0} & \cdots & \mathbf{p}\left(\gamma_{K}, \eta_{K}\right)
\end{array}\right], \\
\mathbf{p}\left(\gamma_{i}, \eta_{i}\right)=\left[\sin \gamma_{i} e^{j \eta_{i}}, \cos \gamma_{i}\right]^{T}, \quad i=1, \cdots, K,
\end{gathered}
$$

where $\gamma_{i}$ and $\eta_{i}$ are the polarization parameters of the $i$ th incoming signal. $\gamma \in[0, \pi / 2]$ and $\eta \in[-\pi, \pi]$ are defined to characterize the respective amplitude ratio and the phase difference between the two polarization components, respectively.

Combining formulas (17)-(19), the following equation can be derived

$$
\begin{aligned}
\mathbf{x}(t)= & {\left[\widetilde{\mathbf{a}}_{\theta}\left(\theta_{1}, \phi_{1}\right), \widetilde{\mathbf{a}}_{\phi}\left(\theta_{1}, \phi_{1}\right), \cdots, \widetilde{\mathbf{a}}_{\theta}\left(\theta_{K}, \phi_{K}\right), \widetilde{\mathbf{a}}_{\phi}\left(\theta_{K}, \phi_{K}\right)\right] } \\
& {\left[\begin{array}{ccc}
\mathbf{p}\left(\gamma_{1}, \eta_{1}\right) & \cdots & \mathbf{0} \\
\vdots & \ddots & \vdots \\
\mathbf{0} & \cdots & \mathbf{p}\left(\gamma_{K}, \eta_{K}\right)
\end{array}\right] \cdot\left[\begin{array}{c}
s_{1}(t) \\
\vdots \\
s_{K}(t)
\end{array}\right]+\mathbf{n}(t) . }
\end{aligned}
$$

The equivalent array manifold of the dual-polarized conformal array is a matrix of $2 N \times 2 K$, and it can be written as

$$
\widetilde{\mathbf{A}}=\left[\widetilde{\mathbf{a}}_{\theta}\left(\theta_{1}, \phi_{1}\right), \widetilde{\mathbf{a}}_{\phi}\left(\theta_{1}, \phi_{1}\right), \cdots, \widetilde{\mathbf{a}}_{\theta}\left(\theta_{K}, \phi_{K}\right), \widetilde{\mathbf{a}}_{\phi}\left(\theta_{K}, \phi_{K}\right)\right] .
$$

Finally, the received data of array can be written as

$$
\begin{aligned}
& \mathbf{x}(t)=\widetilde{\operatorname{APs}}(t)+\mathbf{n}(t), \\
& \mathbf{s}(t)=\left[s_{1}(t), \cdots, s_{K}(t)\right]^{T}, \\
& \mathbf{n}(t)=\left[n_{1 \theta}(t), n_{1 \phi}(t), \cdots, n_{N \theta}(t), n_{N \phi}(t)\right]^{T},
\end{aligned}
$$

where $\mathbf{s}(t)$ denotes the $K \times 1$ vectors of the incident signals, and $\mathbf{n}(t)$ denotes the $2 N \times 1$ Gaussian white noise with zero mean and variance $\sigma_{n}^{2}$. From equation (22), it can be found that all the information of polarization is contained in the matrix $\mathbf{P}$, which can be seen as the coherence coefficient matrix in this model.

\section{Polarization and DOA Estimation}

In Section 2, each polarization signal is decomposed into two orthogonal polarization components, which are considered to be coherent signals coming from the same direction but different polarization. The polarization parameters are modeled as coherence coefficients of each pair of polarization components. And in this section, the decoherence of conformal array is achieved by using the virtual projection transformation and spatial smoothing technique. The DP-MUSIC algorithm is used to estimate the DOA of dual-polarized conformal array.

3.1. Virtual Projection Transformation. Assume that the observation areas of elevation and azimuth angles of the virtual transformation are $\Theta=\left[\theta_{l}, \theta_{r}\right]$ and $\Phi=\left[\phi_{l}, \phi_{r}\right]$, respectively. The areas are evenly divided by intervals $\Delta \theta$ and $\Delta \phi$. In that way, the original array manifold matrix and the virtual array manifold matrix of the dual-polarized uniform planar array can be written as [17]

$$
\begin{aligned}
\widetilde{\mathbf{A}}_{r}= & {\left[\widetilde{\mathbf{a}}_{\theta}\left(\theta_{l}, \phi_{l}\right), \widetilde{\mathbf{a}}_{\phi}\left(\theta_{l}, \phi_{l}\right), \widetilde{\mathbf{a}}_{\theta}\left(\theta_{l}+\Delta \theta, \phi_{l}\right), \cdots,\right.} \\
& \left.\widetilde{\mathbf{a}}_{\phi}\left(\theta_{r}, \phi_{r}-\Delta \phi\right), \widetilde{\mathbf{a}}_{\theta}\left(\theta_{r}, \phi_{r}\right), \widetilde{\mathbf{a}}_{\phi}\left(\theta_{r}, \phi_{r}\right)\right], \\
\mathbf{A}_{v}= & {\left[\mathbf{a}_{p}\left(\theta_{l}, \phi_{l}\right), \mathbf{a}_{p}\left(\theta_{l}+\Delta \theta, \phi_{l}\right), \cdots,\right.} \\
& \left.\mathbf{a}_{p}\left(\theta_{r}, \phi_{r}-\Delta \phi\right), \mathbf{a}_{p}\left(\theta_{r}, \phi_{r}\right)\right] \otimes \mathbf{I}_{2},
\end{aligned}
$$

where $\mathbf{a}_{p}(\theta, \phi)$ is the steering vector of the single-polarized uniform planar array. The approximate transformation can be realized by the virtual transformation relationship between the original array and the virtual array.

$$
\mathbf{A}_{v}=\mathbf{B}^{H} \widetilde{\mathbf{A}}_{r}
$$

where the symbol " $[\cdot]^{H \text { " }}$ denotes the conjugate transpose of the matrix and $\mathbf{B}$ is the $2 N \times 2 N$ transformation array, which can be solved by least squares.

$$
\mathbf{B}=\left(\widetilde{\mathbf{A}}_{r} \widetilde{\mathbf{A}}_{r}^{H}\right)^{-1} \widetilde{\mathbf{A}}_{r} \mathbf{A}_{v}^{H}
$$

The covariance matrix of signal-received data can be written as

$$
\mathbf{R}_{x}=E\left\{\mathbf{x}(t) \mathbf{x}^{H}(t)\right\}=\widetilde{\mathbf{A}} \mathbf{R}_{s} \widetilde{\mathbf{A}}^{H}+\sigma_{n}^{2} \mathbf{I},
$$

where $\mathbf{R}_{s}=\mathbf{P} \widetilde{\mathbf{R}}_{s} \mathbf{P}^{H}$. $\widetilde{\mathbf{R}}_{s}$ is the covariance matrix of signal source, $\sigma_{n}^{2}$ is the noise power, and $\mathbf{I}$ is a $2 \mathrm{~N} \times 2 \mathrm{~N}$ identity matrix. Then, the covariance matrix of the virtual array can be expressed as follows [18]:

$$
\begin{aligned}
\overline{\mathbf{R}}_{x} & =\mathbf{B}^{H} \mathbf{R}_{x} \mathbf{B} \\
& =\mathbf{B}^{H} \widetilde{\mathbf{A}} \mathbf{R}_{s}\left(\mathbf{B}^{H} \widetilde{\mathbf{A}}\right)^{H}+\sigma_{n}^{2} \mathbf{B}^{H} \mathbf{B} .
\end{aligned}
$$

Generally, $\mathbf{B}^{H} \mathbf{B} \neq \mathbf{I}$. Therefore, the white noise of the original array becomes colored noise by the virtual transformation. In order to turn colored noise into white noise, the transformation array $\mathbf{B}$ by prewhitening processing can be changed to

$$
\overline{\mathbf{B}}=\left(\mathbf{B}^{H} \mathbf{B}\right)^{-1 / 2} \mathbf{B}^{H}
$$

Consequently, the covariance matrix of the virtual transformation array after noise prewhitening processing is

$$
\overline{\mathbf{R}}_{x}=\overline{\mathbf{B}} \mathbf{R}_{x} \overline{\mathbf{B}}^{H} \text {. }
$$

As can be seen from the model in equation (22), there are two coherent signals in each group. The spatial forwardbackward smoothing process is used to realize decoherence. The covariance matrix can be expressed as

$$
\overline{\mathbf{R}}_{x}^{f b}=\frac{\left(\overline{\mathbf{R}}_{x}+\mathbf{J} \overline{\mathbf{R}}_{x}^{*} \mathbf{J}\right)}{2},
$$

where $\mathbf{J}$ is the $2 N \times 2 N$ inverse identity matrix, and the symbol " $(\cdot)^{*}$ ” denotes complex conjugate of matrix. 
3.2. Parameter Estimation. After the above processing, the depolarization and decoherence are realized. In this paper, the DP-MUSIC algorithm is used to estimate the azimuth and elevation of signals.

The eigenvalue decomposition (EVD) of $\overline{\mathbf{R}}_{x}^{f b}$

$$
\overline{\mathbf{R}}_{x}^{f b}=\overline{\mathbf{U}}_{s} \overline{\boldsymbol{\Lambda}}_{\mathrm{s}} \overline{\mathbf{U}}_{s}+\overline{\mathbf{U}}_{n} \overline{\boldsymbol{\Lambda}}_{n} \overline{\mathbf{U}}_{n}
$$

where $\overline{\mathbf{U}}_{s} \in \mathbb{C}^{2 N \times 2 K}$ and $\overline{\mathbf{U}}_{n} \in \mathbb{C}^{2 N \times(2 N-2 K)}$ are the signal and noise subspaces spanned by eigenvectors corresponding to the eigenvalues $\bar{\Lambda}_{s}$ and $\bar{\Lambda}_{n}$, respectively.

The steering vector of the virtual projection is $\overline{\mathbf{B}} \widetilde{\mathbf{a}}(\theta, \phi)$. Define

$$
\begin{aligned}
\mathbf{Q}(\theta, \phi) & =\widetilde{\mathbf{a}}^{H}(\theta, \phi) \overline{\mathbf{B}}^{H} \overline{\mathbf{U}}_{n} \overline{\mathbf{U}}_{n}^{H} \overline{\mathbf{B}} \widetilde{\mathbf{a}}(\theta, \phi) \\
& =\left[\widetilde{\mathbf{a}}_{\theta}(\theta, \phi), \widetilde{\mathbf{a}}_{\phi}(\theta, \phi)\right]^{H} \overline{\mathbf{B}}^{H} \overline{\mathbf{U}}_{n} \overline{\mathbf{U}}_{n}^{H} \overline{\mathbf{B}}\left[\widetilde{\mathbf{a}}_{\theta}(\theta, \phi), \widetilde{\mathbf{a}}_{\phi}(\theta, \phi)\right],
\end{aligned}
$$

where $\mathbf{Q}(\theta, \phi)$ is a $2 \times 2$ matrix. The arbitrary polarization signal has at least one component in the polarization space. Then when $(\theta, \phi)$ is equal to the actual arrival directions $\left(\theta_{i}, \phi_{i}\right), i=1, \cdots, K$, the diagonal elements in the $\mathbf{Q}(\theta, \phi)$ has at least one element that is zero. $\overline{\mathbf{B}}^{H} \overline{\mathbf{U}}_{n} \overline{\mathbf{U}}_{n}{ }^{H} \overline{\mathbf{B}}$ is a positive semidefinite matrix, so the following pseudospectral can be constructed to estimate the angles of the signals:

$$
P(\theta, \phi)=\frac{1}{\min _{1 \leq i \leq 2} \mathbf{Q}_{i i}(\theta, \phi)}
$$

The DOAs are estimated by searching the $K$ highest peaks of the pseudospectrum, denoted as $\left(\widehat{\theta}_{i}, \widehat{\phi}_{i}\right)$.

After DOA estimated values are obtained, the polarization parameters are estimated by using DOA estimation values and original received data. $\mathbf{U}_{s}$ and $\mathbf{U}_{n}$ are the signal and noise subspaces of $\mathbf{R}_{x}$. The DOA estimation value for each group corresponds to the estimates of one group of polarization parameters. Plugging the estimated values $\left(\widehat{\theta}_{i}, \widehat{\phi}_{i}\right)$ into formula (22), and the polarized steering vector can be expressed as

$$
\mathbf{a}(\widehat{\theta}, \widehat{\phi}, \gamma, \eta)=\widetilde{\mathbf{a}}(\widehat{\theta}, \widehat{\phi}) \mathbf{p}(\gamma, \eta) .
$$

Each set of polarization parameters matches the corresponding angle-estimated value. The estimation values of the polarization parameters can be obtained from the following formula:

$$
\left(\widehat{\gamma}_{i}, \widehat{\eta}_{i}\right)=\underset{(\gamma, \eta)}{\arg \max } \frac{1}{\left|\mathbf{a}^{H}\left(\widehat{\theta}_{i}, \widehat{\phi}_{i}, \gamma, \eta\right) \mathbf{U}_{n} \mathbf{U}_{n}^{H} \mathbf{a}\left(\widehat{\theta}_{i}, \widehat{\phi}_{i}, \gamma, \eta\right)\right|},
$$

where $i=1, \cdots, K$. The DOA is estimated on the basis of the virtual projection, which will lead to a transformation error. In order to reduce the error of the DOA estimation caused by the virtual projection transformation, the DOAs are reestimated after obtaining the estimation values of the polarization parameters.

The estimated values $\left(\widehat{\gamma}_{i}, \widehat{\eta}_{i}\right)$ take the place of polarization parameters $(\gamma, \eta)$ in formula $(22)$, and the polarized steering vector can be rewritten as

$$
\mathbf{a}(\theta, \phi, \widehat{\gamma}, \widehat{\eta})=\widetilde{\mathbf{a}}(\theta, \phi) \mathbf{p}(\widehat{\gamma}, \widehat{\eta}) .
$$

The pseudospectral expression of DOAs refined estimation is shown as

$$
P(\theta, \phi)=\sum_{i=1}^{K} \frac{1}{\left|\mathbf{a}^{H}\left(\theta, \phi, \widehat{\gamma}_{i}, \widehat{\eta}_{i}\right) \mathbf{U}_{n} \mathbf{U}_{n}^{H} \mathbf{a}\left(\theta, \phi, \widehat{\gamma}_{i}, \widehat{\eta}_{i}\right)\right|} .
$$

From formula (39), the further refined estimation of DOAs can be obtained.

\section{Cramer-Rao Bound}

The CRB determines the lower bound on the variances of unbiased parameters estimation. In order to measure the performance of the DP-MUSIC algorithm, the estimation $\mathrm{CRB}$ for the dual-polarized conformal array is derived here.

The signal model expressed in formula (22) should be converted into a discrete time digital domain, and the probability distribution function (PDF) of $L$ samples is [19]

$$
\begin{aligned}
p(\mathbf{x} \mid \theta, \phi, \gamma, \eta)= & \frac{1}{\left(2 \pi \sigma_{n}^{2}\right)^{L / 2}} \\
& \cdot \exp \left\{-\frac{1}{\sigma_{n}^{2}} \cdot \sum_{n=0}^{L-1}[\mathbf{x}(n)-\mathbf{A}(\theta, \phi, \gamma, \eta) \mathbf{s}(n)]^{H}\right. \\
& \cdot[\mathbf{x}(n)-\mathbf{A}(\theta, \phi, \gamma, \eta) \mathbf{s}(n)]\} .
\end{aligned}
$$

The array covariance matrix $\mathbf{R}_{x}$ contains $4 K$ unknown parameters, they are $K$ azimuth angles, $K$ elevation angles, and $2 K$ polarization parameters. Define the unknown parameter vector embedded in $\mathbf{R}_{x}$ as $\boldsymbol{\xi}=[\boldsymbol{\theta}, \boldsymbol{\phi}, \boldsymbol{\gamma}, \boldsymbol{\eta}]^{T}$, where $\boldsymbol{\theta}=\left[\theta_{1}, \cdots, \theta_{K}\right], \quad \boldsymbol{\phi}=\left[\phi_{1}, \cdots, \phi_{K}\right], \quad \boldsymbol{\gamma}=\left[\gamma_{1}, \cdots, \gamma_{K}\right], \quad$ and $\boldsymbol{\eta}=\left[\eta_{1}, \cdots, \eta_{K}\right]$. Each element of the Fisher information matrix entry can be calculated by using the following formula:

$$
\mathbf{F}_{i j}=-E\left\{\frac{\partial^{2} \ln (p(\mathbf{x} \mid \theta, \phi, \gamma, \eta))}{\partial \boldsymbol{\xi}_{i} \partial \boldsymbol{\xi}_{j}}\right\}, \quad i, j=1, \cdots, 4 K .
$$

Consequently, the $4 K \times 4 K$ Fisher information matrix can be written as

$$
\mathbf{F}=\left[\begin{array}{llll}
\mathbf{F}_{\theta \theta} & \mathbf{F}_{\theta \phi} & \mathbf{F}_{\theta \gamma} & \mathbf{F}_{\theta \eta} \\
\mathbf{F}_{\phi \theta} & \mathbf{F}_{\phi \phi} & \mathbf{F}_{\phi \gamma} & \mathbf{F}_{\phi \eta} \\
\mathbf{F}_{\gamma \theta} & \mathbf{F}_{\gamma \phi} & \mathbf{F}_{\gamma \gamma} & \mathbf{F}_{\gamma \eta} \\
\mathbf{F}_{\eta \theta} & \mathbf{F}_{\eta \phi} & \mathbf{F}_{\eta \gamma} & \mathbf{F}_{\eta \eta}
\end{array}\right],
$$

where $\mathbf{F}_{\theta \theta}$ and $\mathbf{F}_{\phi \phi}$ refer to the elevation and azimuth estimation block, respectively. $\mathbf{F}_{\gamma \gamma}$ and $\mathbf{F}_{\eta \eta}$ denote the polarization parameters estimation block, the rest of modules are the cross-correlation estimation block of relevant parameters.

Combining formulas (40) and (41), the following equation can be further derived: 


$$
\mathbf{F}_{i j}=\mathrm{L} \cdot \mathrm{SNR} \cdot\left(\frac{\partial \mathbf{A}^{H}}{\partial \xi_{i}} \frac{\partial \mathbf{A}}{\partial \xi_{j}}+\frac{\partial \mathbf{A}^{H}}{\partial \boldsymbol{\xi}_{i}} \frac{\partial \mathbf{A}}{\partial \boldsymbol{\xi}_{j}}\right),
$$

where $\mathrm{SNR}=\sigma_{s}^{2} / \sigma_{n}^{2}$ and $E\left[s^{2}(n)\right]=\sigma_{s}^{2}$. The parameter $\xi$ contains angle parameters and polarization parameters, and their partial derivatives can be calculated by the following formulas:

$$
\left\{\begin{array}{l}
\frac{\partial \mathbf{A}}{\partial \theta_{i}}=\frac{\partial \tilde{\mathbf{A}}}{\partial \theta_{i}} \mathbf{P} \\
\frac{\partial \mathbf{A}}{\partial \phi_{i}}=\frac{\partial \tilde{\mathbf{A}}}{\partial \phi_{i}} \mathbf{P} \\
\frac{\partial \mathbf{A}}{\partial \gamma_{i}}=\tilde{\mathbf{A}} \frac{\partial \mathbf{P}}{\partial \gamma_{i}} \\
\frac{\partial \mathbf{A}}{\partial \eta_{i}}=\tilde{\mathbf{A}} \frac{\partial \mathbf{P}}{\partial \eta_{i}}
\end{array}\right.
$$

where

$$
\begin{aligned}
\frac{\partial \tilde{\mathbf{A}}(\theta, \phi)}{\partial \theta_{i}}= & \mathbf{G} \frac{\partial\left(\mathbf{T}(\theta, \phi) \cdot\left(\mathbf{a}(\theta, \phi) \otimes \mathbf{I}_{2}\right)\right)}{\partial \theta_{i}}, \\
\frac{\partial\left(\mathbf{T}(\theta, \phi) \cdot\left(\mathbf{a}(\theta, \phi) \otimes \mathbf{I}_{2}\right)\right)}{\partial \theta_{i}}= & \frac{\partial \mathbf{T}(\theta, \phi)}{\partial \theta_{i}} \cdot\left(\mathbf{a}(\theta, \phi) \otimes \mathbf{I}_{2}\right) \\
& +\mathbf{T}(\theta, \phi) \cdot \frac{\partial\left(\mathbf{a}(\theta, \phi) \otimes \mathbf{I}_{2}\right)}{\partial \theta_{i}} \\
\frac{\partial \mathbf{T}(\theta, \phi)}{\partial \theta_{i}}= & \frac{\partial \mathbf{T}_{c s}\left(\theta^{\prime}, \phi^{\prime}\right)}{\partial \theta_{i}} \mathbf{R}_{g l} \mathbf{T}_{s c}(\theta, \phi) \\
& +\mathbf{T}_{c s}\left(\theta^{\prime}, \phi^{\prime}\right) \mathbf{R}_{g l} \frac{\partial \mathbf{T}_{s c}(\theta, \phi)}{\partial \theta_{i}}
\end{aligned}
$$

The partial derivative of parameters $\phi, \gamma$, and $\eta$ can be obtained by a similar derivation process. The CRB can be obtained from the inverse of the Fisher information matrix. Define $\mathbf{G}=\mathbf{F}^{-1}$, the CRB can be calculated by the following formula:

$$
\begin{aligned}
& \mathrm{CRB}_{\mathbf{a}}=\sqrt{\frac{1}{2 K} \sum_{i=1}^{2 K} \mathbf{G}_{i i},} \\
& \mathrm{CRB}_{\gamma}=\sqrt{\frac{1}{K} \sum_{i=2 K+1}^{3 K} \mathbf{G}_{i i},} \\
& \mathrm{CRB}_{\eta}=\sqrt{\frac{1}{K} \sum_{i=3 K+1}^{4 K} \mathbf{G}_{i i},}
\end{aligned}
$$

where $\mathrm{CRB}_{\mathrm{a}}$ is the Cramer-Rao bound for the DOA. CRB and $\mathrm{CRB}_{\eta}$ are the Cramer-Rao bound for polarization parameter.

\section{Simulation Results}

Without loss of generality, an arc conformal array with $N=15$ elements is considered as shown in Figure 2. The central angle of the arc is $150^{\circ}$. The antenna element adopts patch antenna model of dual-polarized, and its pattern is [20]

$$
\left\{\begin{array}{l}
\mathrm{g}_{\theta}\left(\theta^{\prime}, \phi^{\prime}\right)=\left[J_{2}\left(\pi d \sin \theta^{\prime} / \lambda\right)-J_{0}\left(\pi d \sin \theta^{\prime} / \lambda\right)\right] \\
\cdot\left[\cos \phi^{\prime}-j \sin \phi^{\prime}\right], 0 \leq \theta^{\prime} \leq \pi / 2, \\
\mathrm{~g}_{\phi}\left(\theta^{\prime}, \phi^{\prime}\right)=\left[J_{2}\left(\pi d \sin \theta^{\prime} / \lambda\right)+J_{0}\left(\pi d \sin \theta^{\prime} / \lambda\right)\right] \\
\cdot \cos \theta^{\prime}\left[\sin \phi^{\prime}-j \cos \phi^{\prime}\right], 0 \leq \theta^{\prime} \leq \pi / 2, \\
\mathrm{~g}_{\theta}\left(\theta^{\prime}, \phi^{\prime}\right)=\mathrm{g}_{\phi}\left(\theta^{\prime}, \phi^{\prime}\right)=0, \theta^{\prime}>\pi / 2,
\end{array}\right.
$$

where $J_{0}$ and $J_{2}$ are the first kind of zero-order and secondorder Bessel functions, respectively. $d=\lambda / 2$ is the distance between adjacent elements.

In the first simulation, all the signals are assumed to come from the XOY plane. There are $K=3$ incident signals with the same power, and the angles of signals are $\left(\theta_{1}, \phi_{1}\right)=\left(90^{\circ},-40^{\circ}\right),\left(\theta_{2}, \phi_{2}\right)=\left(90^{\circ}, 15^{\circ}\right)$, and $\left(\theta_{3}, \phi_{3}\right)=$ $\left(90^{\circ}, 40^{\circ}\right)$, respectively. The azimuth scans range from $-70^{\circ}$ to $70^{\circ}$ with the step of $0.1^{\circ}$, the signal-to-noise radio (SNR) is $10 \mathrm{~dB}$, and the number of snapshots are $L=200$. The polarization state angles are $\left(\gamma_{1}, \eta_{1}\right)=\left(20^{\circ}, 60^{\circ}\right),\left(\gamma_{2}, \eta_{2}\right)=$ $\left(30^{\circ}, 45^{\circ}\right)$, and $\left(\gamma_{3}, \eta_{3}\right)=\left(60^{\circ}, 30^{\circ}\right)$, respectively.

The pseudospectrum is shown in Figure 3. Compared with polarization smoothing and initial estimation, the DOA refined estimation has sharper peaks, and it has higher accuracy. It can be seen that the PSA has failed and is not applicable to the dual-polarized conformal array. The estimation values of polarization parameters are obtained by formula (37), and the corresponding spectral peak diagrams are shown in Figures 4, 5, and 6, respectively.

The Monte Carlo experiments are used to verify the estimated performance of the proposed method. The root mean square error (RMSE) of the DOA and polarization parameters are defined as

$$
\begin{aligned}
& \operatorname{RMSE}_{\mathbf{a}}=\frac{1}{\sqrt{M K}}\left[\sum_{n=1}^{M}\left[\sum_{i=1}^{K}\left(\theta_{i}-\widehat{\theta}_{i, n}\right)+\left(\phi_{i}-\widehat{\phi}_{i, n}\right)\right]\right]^{1 / 2}, \\
& \operatorname{RMSE}_{\gamma}=\frac{1}{\sqrt{M K}}\left[\sum_{n=1}^{M}\left[\sum_{i=1}^{K}\left(\gamma_{i}-\widehat{\gamma}_{i, n}\right)\right]\right]^{1 / 2}, \\
& \operatorname{RMSE}_{\eta}=\frac{1}{\sqrt{M K}}\left[\sum_{n=1}^{M}\left[\sum_{i=1}^{K}\left(\eta_{i}-\widehat{\eta}_{i, n}\right)\right]\right]^{1 / 2},
\end{aligned}
$$




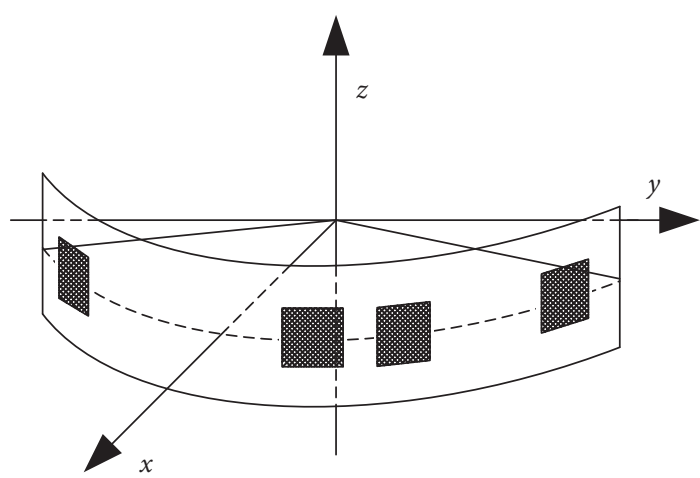

Figure 2: Arc conformal array model.

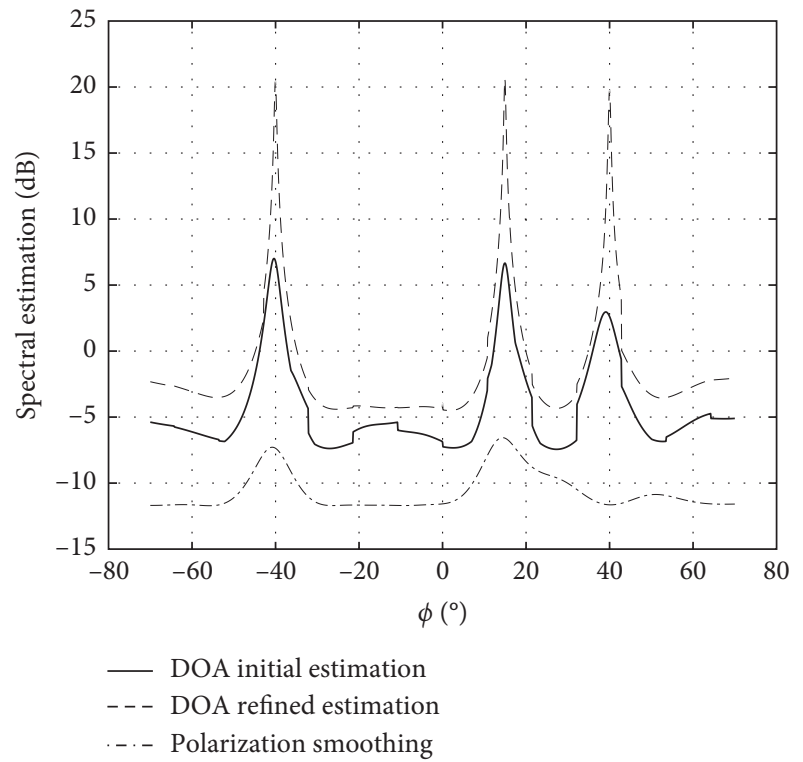

Figure 3: The DOA estimation.

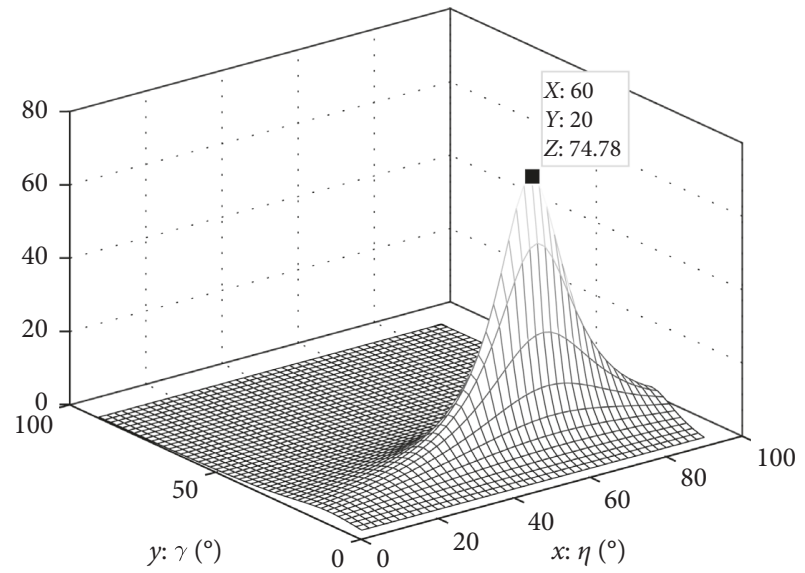

Figure 4: Polarization estimation result with $(\theta, \phi)=\left(90^{\circ},-40^{\circ}\right)$.

where $\widehat{\theta}_{i, n}, \widehat{\phi}_{i, n}$ and $\widehat{\gamma}_{i, n}, \widehat{\eta}_{i, n}$ refer to the DOA estimated value and polarization parameters estimated value of the $i$ th signal in the $n$th Monte Carlo experiment, respectively. $M=100$ is the number of Monte Carlo experiment. The RMSE is

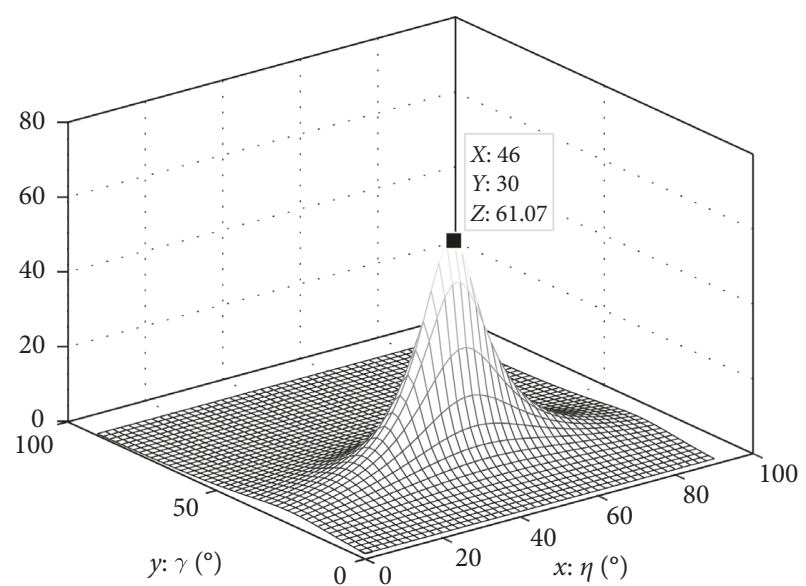

Figure 5: Polarization estimation result with $(\theta, \phi)=\left(90^{\circ}, 15^{\circ}\right)$.

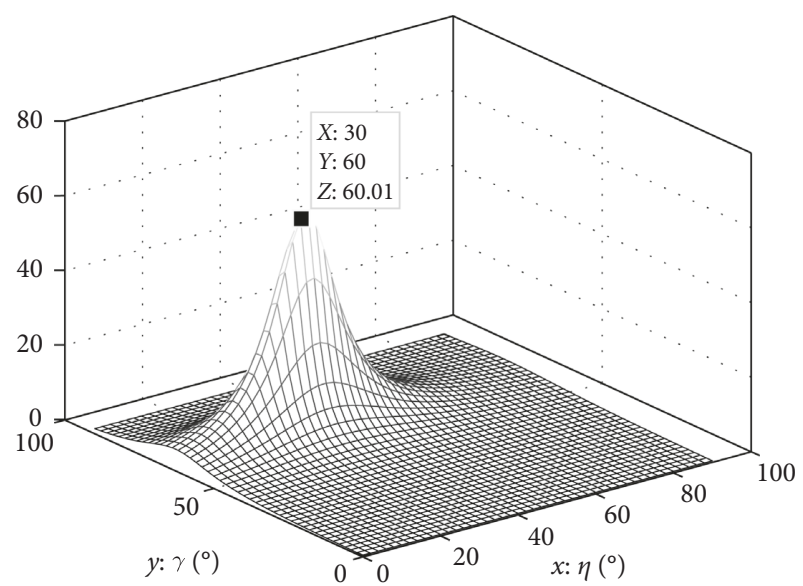

Figure 6: Polarization estimation result with $(\theta, \phi)=\left(90^{\circ}, 40^{\circ}\right)$.

compared with $\mathrm{CRB}$ to evaluate the performance of parameters estimation in the following simulations.

In the second simulation, the SNR changes from $-5 \mathrm{~dB}$ to $15 \mathrm{~dB}$ with the step of $2 \mathrm{~dB}$. The other conditions are the same as the first simulation. Figure 7 shows that the RMSE performance of azimuth refined estimation is close to the CRB. Figure 8 shows that corresponding RMSE to the polarization parameters for SNR from $-5 \mathrm{~dB}$ to $15 \mathrm{~dB}$ with the step $2 \mathrm{~dB}$.

In the third simulation, the number of snapshots change from 100 to 1000 with the step of 100 . The SNR is fixed at $0 \mathrm{~dB}$ and the other conditions are the same as the first simulation. With the increases of snapshots, Figure 9 illustrates the RMSE performance versus the number of snapshots. The RMSE performance of refined estimation is significantly better than the initial estimation. It can be seen from Figure 10 that as the number of snapshots increases, the RMSE of each curve shows a downward trend.

According to Figures 7 and 9, the RMSE of DOA initial estimation value and refined estimation value decreases with the increase of the SNR and snapshots, and the error curve of refined estimation approaches the lower bound. Figures 8 and 10 show that the comparison of RMSE performance and 


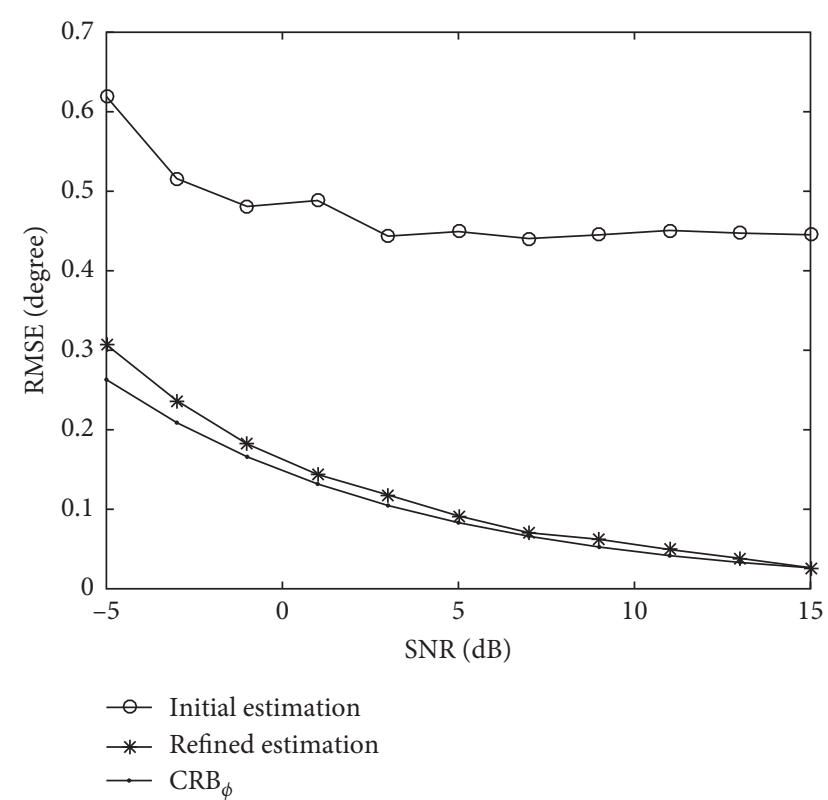

Figure 7: Angle estimation performances with different SNR.

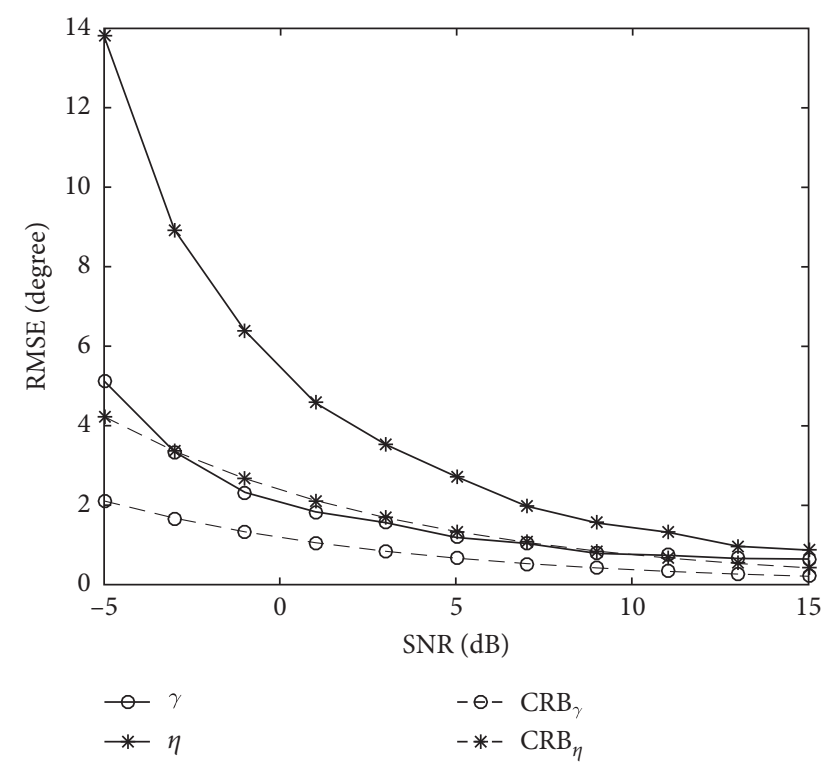

FIGURe 8: Polarization parameters estimation performances with different SNR.

CRB for polarization parameters. Through the comparison of above simulation results, it can be seen that the error of angle estimation is smaller than that of polarization parameter estimation. Since there are only two receiving components in the polarization domain while there are $\mathrm{N}$ receivers in the space domain, it is reasonable that the estimation performance in the spatial domain is better than the polarization domain. However, the RMSE of the polarization parameters still have a good performance when the SNR and the number of snapshots are large.

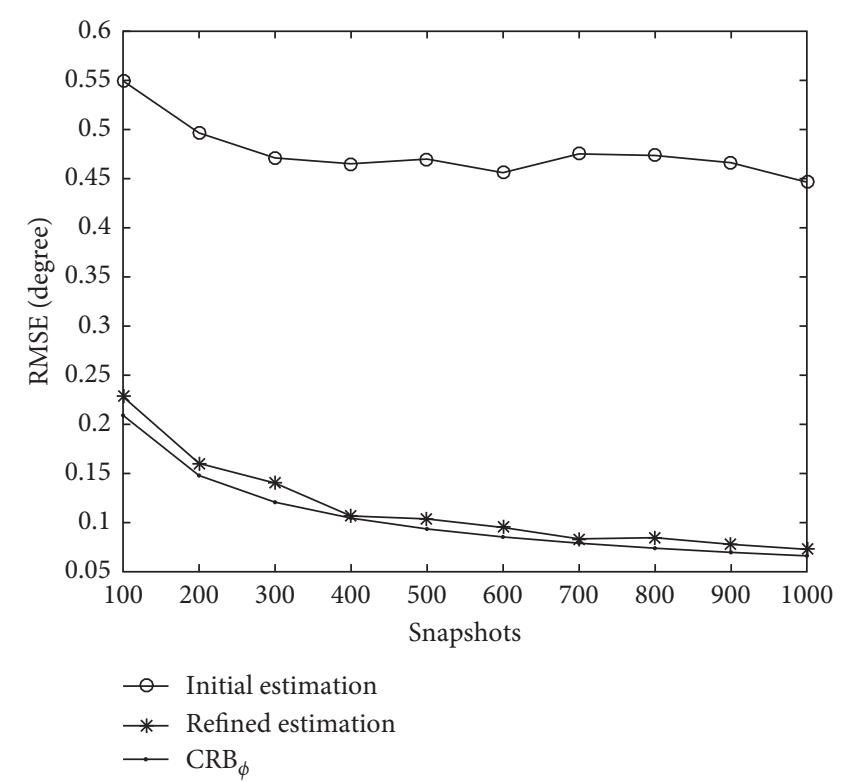

FIGURE 9: Angle estimation performances with different snapshots.

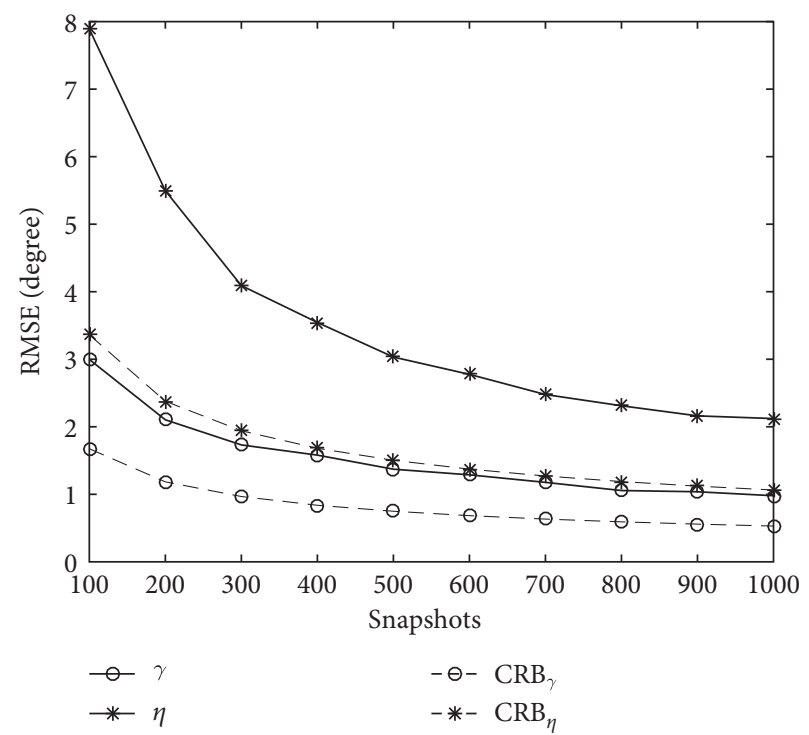

FIgURE 10: Polarization parameters estimation performances with different snapshots.

\section{Conclusion}

This paper presents the DP-MUSIC algorithm to estimate the polarization and DOA based on the dual-polarized conformal array. Each polarization signal is decomposed into two orthogonal polarization components, which are considered to be a pair of coherent signals. The polarization parameters are modeled as the equivalent coherence coefficients of the orthogonal polarization components. Then, the decoherence method can be used to separate the DOA information coupled with the polarization information. Consequently, the polarization parameters and DOA can be estimated separately by the proposed algorithm. Moreover, 
the DOA is re-estimated to further improve the DOA estimation accuracy. The DP-MUSIC algorithm can achieve the DOA and polarization estimation for arbitrary polarization signals of the dual-polarized conformal array. Finally, the CRB is derived and simulation results demonstrate the effectiveness of the proposed algorithm.

\section{Data Availability}

All the data used in the manuscript is generated by simulations. The data used to support the findings of this study is available from the corresponding author upon request.

\section{Conflicts of Interest}

The authors declare that there are no conflicts of interest regarding the publication of this paper.

\section{Acknowledgments}

This work is supported by the the Natural Science Foundation of Anhui Province (no. 1608085MF134).

\section{References}

[1] L. Josefsson and P. Persson, Conformal Array Antenna Theory and Design, IEEE Press, Piscataway, NJ, USA, 2006.

[2] R. Schmidt, "Multiple emitter location and signal parameter estimation," IEEE Transactions on Antennas and Propagation, vol. 34 , no. 3, pp. 276-280, 1986.

[3] A. Tanaka and H. Imai, "Music-based DOA estimation by oblique projection along the signal subspace," in Proceedings of the IEEE Workshop on Statistical Signal Processing (SSP), pp. 300-303, Gold Coast, VIC, Australia, July 2014.

[4] A. Liu, Q. Yang, X. Zhang et al., "Direction-of-arrival estimation for coprime array using compressive sensing based array interpolation," International Journal of Antennas and Propagation, vol. 2017, Article ID 6425067, 10 pages, 2017.

[5] J. Li and R. T. Compton, "Angle and polarization estimation using esprit with a polarization sensitive array," IEEE Transactions on Antennas and Propagation, vol. 39, no. 9, pp. 1376-1383, 1991

[6] D. Rahamim, J. Tabrikian, and R. Shavit, "Source localization using vector sensor array in a multipath environment," IEEE Transactions on Signal Processing, vol. 52, no. 11, pp. 30963103, 2004.

[7] G. Zheng and B. Wu, "Polarisation smoothing for coherent source direction finding with multiple-input and multipleoutput electromagnetic vector sensor array," IET Signal Processing, vol. 10, no. 8, pp. 873-879, 2016.

[8] Z. Qi, Y. Guo, B. Wang, and C. Gong, "DOA estimation algorithm for conical conformal array antenna," in Proceedings of the International Radar Conference, pp. 1-4, Guilin, China, April 2009.

[9] Z.-s. Qi, Y. Guo, and B.-h. Wang, "Blind direction-of-arrival estimation algorithm for conformal array antenna with respect to polarisation diversity," IET Microwaves, Antennas \& Propagation, vol. 5, no. 4, pp. 433-442, 2011.

[10] B. Obeidat, N. Y. Zhang, and M. G. Amin, "Performance analysis of DOA estimation using dual-polarized antenna arrays," in Proceedings of the IEEE International Symposium on Signal Processing and Information Technology, pp. 423-428, Athens, Greece, December 2005.
[11] M. G. Pralon, L. G. Pralon, D. Neudert-Schulz, and R. S. Thoma, "On the performance of real dual-polarized antenna arrays for 2D unconditional Direction of Arrival estimation," in Proceedings of the European Conference on Antennas and Propagation, pp. 1-5, Davos, Switzerland, April 2016.

[12] Y. Han, Q. Fang, F. Yan, M. Jin, and X. Qiao, "Joint DOA and polarization estimation for unequal power sources based on reconstructed noise subspace," Journal of Systems Engineering and Electronics, vol. 27, no. 3, pp. 501-513, 2016.

[13] L. Chao and Z. Ding, "Direction of arrival estimation for conformal array with polarization diversity," Chinese Journal of Radio Science, vol. 30, no. 2, pp. 274-281, 2015.

[14] J. Zhang, S. Chai, K. Xiao, L. Ding, and F. Zhao, "Differential feeding technique for full-polarization conformal phased array," in Proceedings of the International Workshop on Electromagnetics: Applications and Student Innovation Competition, pp. 87-88, London, UK, June 2017.

[15] T. Milligan, "More applications of Euler rotation angles," IEEE Antennas and Propagation Magazine, vol. 41, no. 4, pp. 78-83, 1999.

[16] C. Liu, Z. Ding, and X. Liu, "A low complexity 2D pattern synthesis algorithm for cylindrical array," International Journal of Antennas and Propagation, vol. 2013, Article ID 352843, 6 pages, 2013.

[17] Q. Li, Y. Jiang, and X. Diao, “A DOA estimation algorithm of virtual array based on beam forming," in Proceedings of the International Conference on Electrical and Control Engineering (ICECE), pp. 945-948, Yichang, China, September 2011.

[18] X. Chen, J. Xin, N. Zheng, and A. Sano, "Direction-of-arrival estimation of coherent narrowband signals with arbitrary linear array," in Proceedings of the IEEE International Workshop on Signal Processing Systems (SiPS), pp. 1-5, Lorient, France, October 2017.

[19] S. Mohammadi, A. Ghani, and S. H. Sedighy, "Direction-ofarrival estimation in conformal microstrip patch array antenna," IEEE Transactions on Antennas and Propagation, vol. 66, no. 1, pp. 511-515, 2018.

[20] J. R. James, P. S. Hall, and C. Wood, Microstrip Antenna: Theory and Design, Peter Peregrinus, London, UK, 1981. 


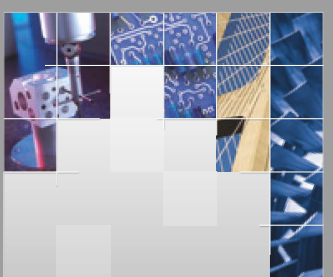

\section{Enfincering}
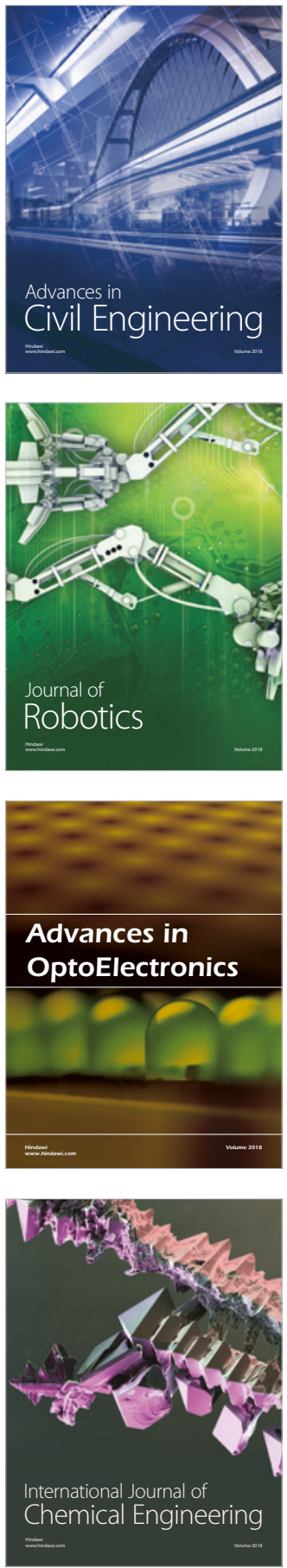

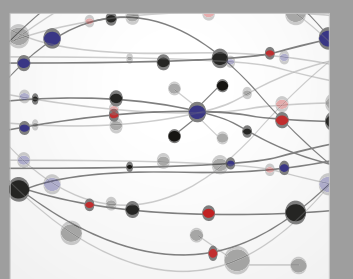

\section{Rotating \\ Machinery}

The Scientific World Journal

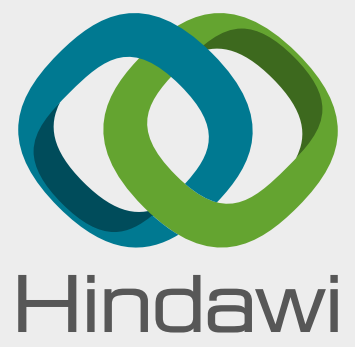

Submit your manuscripts at

www.hindawi.com
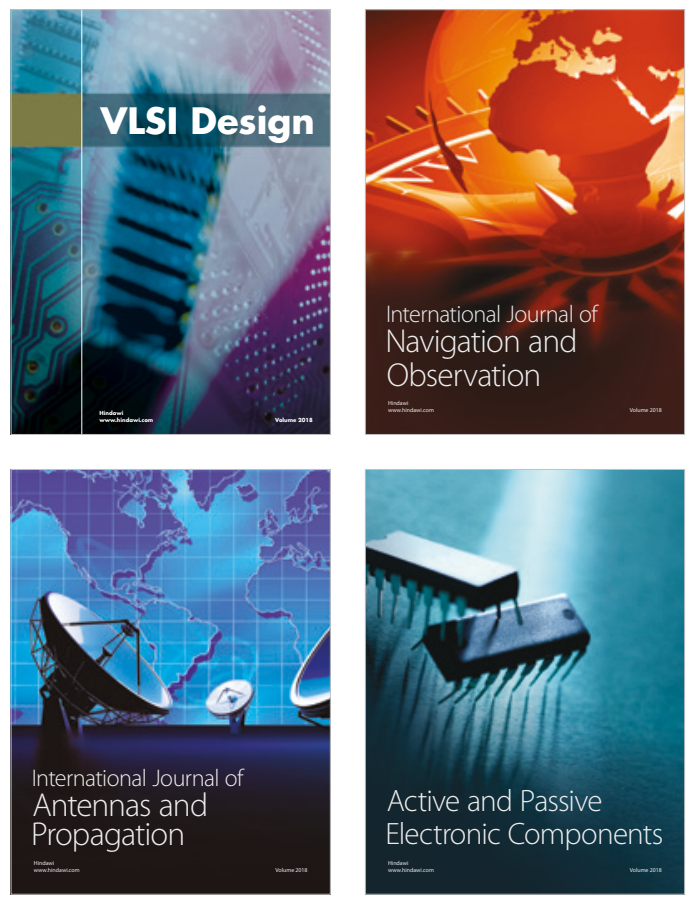
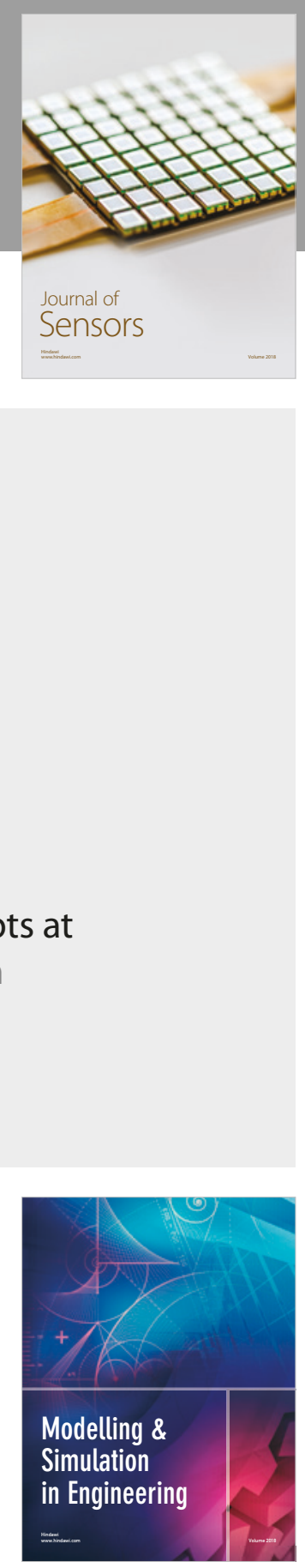

\section{Advances \\ Multimedia}
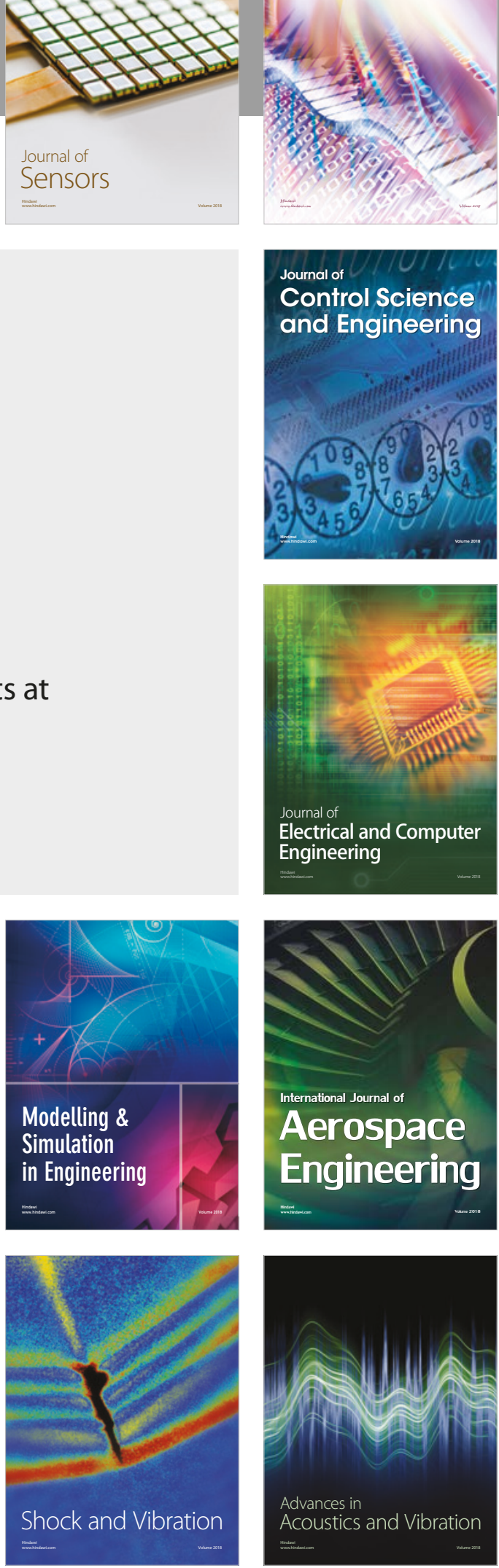